\title{
Impact of Alternaria solani (Early blight) on cultivated tomato (Solanum lycopersicum L.) in North-eastern region of India and identification of early blight disease resistant tomato genotypes
}

\author{
Khaidem Malemnganba Meitei ${ }^{1}$, G.C. Bora ${ }^{1}$, Senjam Jinus Singh ${ }^{2},{ }^{*}$ Anjan Kumar Sinha ${ }^{3}$ \\ ${ }^{1}$ Department of Plant Breeding and Genetics, Assam Agricultural University, Jorhat- 785013 (Assam), INDIA \\ ${ }^{2}$ Department of Vegetable Science, Chaudhary Charan Singh Haryana Agricultural University, Hisar-125004 \\ (Haryana), INDIA \\ ${ }^{3}$ Department of Botany, Bankura Sammilani College, Bankura-722133 (West Bengal), INDIA \\ "Corresponding author. E-mail: anjansinha06@gmail.com \\ Received: January 29, 2015; Revised received: June 22, 2015; Accepted: August 25, 2015
}

\begin{abstract}
The present investigation was carried out to screen genotypes for resistance to Early blight disease of tomato (Solanum lycopersicum L.) of North Eastern region of India. Field trial was conducted in the Experimental Farm, Department of Horticulture, Assam Agricultural University, Jorhat during the years 2012-13 and 2013-14 consecutively. The disease severity of tomato genotypes was assessed by 0-5 points scale, percent Disease Incidence (PDI). Of the total materials screened, Sel-35 (TLBRH-6 X Konbilahi) and Sel-19 (TLBRH-6 X Konbilahi) were highly resistant, 7 were resistant, 14 were moderately resistant, 16 were susceptible and 6 were highly susceptible under field condition after inoculation during both years. The genotype having high yield and resistant to early blight was 10/TOLCVRES-3. The genotypes resistant to early blight but having low yield (Sel-35, Sel-19, Sel-9 and Sel-16) may be utilized in future breeding programme for improving yield through selection for higher fruit weight and fruit diameter. Alternatively, they may be used as parents in hybridization or backcrossing programme in order to transfer the gene for resistance to early blight to already adapted high yield varieties.
\end{abstract}

Keyword: Early blight, North-eastern region, Resistant, Solanum lycopersicum

\section{INTRODUCTION}

Tomato (Solanum lycopersicum L.) [formerly Lycopersicon esculentum Miller] is one of the most significant vegetable crops and cultivated in throughout the globe. In plant breeding study, the main objective of a breeder is to improve the fruit yield, a complex quantitative trait leading continuous variation, especially in major vegetable crops like tomato. Determining the appropriate selection indicia and development of efficient breeding scheme, the studies on genetic parameters and association analysis is much important (Chaerani et al., 2007; Sharma et al., 2008). The maladies leading to various horticultural yield loses in tomato is caused by fungi, bacteria, viruses, nematodes and also abiotic factors (Balanchard, 1992). Globally, early blight caused by the pathogen Alternaria solani (Ellis and Martin) Sorauer, most aggressive and destructive disease (Fry, 2008; Kumar and Srivastava, 2013) is an economically remarkable malady (Peralta et al., 2005; Singh et al., 2013) of cultivated tomato. The different agro-climatic zones suffer with heavy dew (Rotem and Reichert, 1964; Singh et al., 2011), heavy rainfall [Northeast region of India reported the highest rainfall receiving on the earth (Jain et al., 2012 ), high humidity (Sherf ISSN : 0974-9411 (Print), 2231-5209 (Online) All Rights Reserved (C) Applied and Natural Science Foundation www.ansfoundation.org and MacNab, 1986; Singh et al., 2013) and fairly high temperatures $\left(24-29^{\circ} \mathrm{C}\right)$ (Yadav and Singh, 1998; Singh et al., 2013) are more prevalent of this malady. Thus, it leads the most difficult tasks for plant breeder when high temperature and humidity conditions are prevalent. The damages caused by early blight from various parts of the countries viz., India, Canada, United States and Nigeria (Basu, 1974) reported agricultural yield losses up to 79\% (Basu, 1974; Singh, 1985; Datar and Mayee, 1981, Yadav and Dabbas, 2012). In horticultural fruit crops loss may be as high as $95 \%$ under severe epiphytotic condition (Sridha and Naik, 1983). Disease-management strategies mainly depend on chemical fungicide applications, which are uneconomical and less effective due to increasing resistance of the pathogen against fungicides. Thus, identification of resistant sources from wild tomato species may be an effective method of integrated disease management strategy by reducing the environmental pollution by chemical toxicity. Early blight resistance was conferred by recessive polygenes at both seedling and adult plant stages (Thirthammallappa and Lohithaswa, 2000). Many researchers identified the potent resistant to moderate resistant sources mainly in wild species $S$. 
pimpinellifolium L. [formerly L. pimpinellifolium (L.) Mill.] (Kalloo and Banerjee, 1993; Foolad, 2002 and 2005), S. peruvianum L. [formerly L. peruvianum (L.) Mill.] (Chaerani et al., 2007) and S. habrochaites (formerly L. hirsutum Dunal) (Barksdale and Stoner, 1977; Chaerani et al., 2007; Kalloo and Banerjee, 1993; Poysa and Tu, 1997; Foolad et al., 2000; Thirthamalappa and Lohithaswa, 2000; Singh et al., 2013).

Development and screening of early blight-resistant tomato cultivars following appropriate plants breeding tools is the only possible path for the identification and utilization of genetic resources resistant to Alternaria solani (Ellis and Martin) Sorauer in tomato. Although vast genetic diversity exists in well adapted cultivars/ germplasms in tomato in North-eastern region of India, so far not much systematic study on resistance or susceptibility level of existing tomato genetic resources has been conducted. The production of this crop is low in North-eastern states of India, which falls in the highest rainfall receiving regions on the earth (Jain et al., 2012), as compared to the other states of the country. Besides other reasons, the problems associated with lower production are non availability of good varieties and incidence of diseases affecting the crop. Therefore, it is important to study the available genotypes of the crop in order to identify high yielding varieties with desirable characteristics like earliness and resistance to pest and diseases. Considering the points mentioned above, a study for finding out the extent of genetic variability for yield and resistance to early blight in tomato (Solanum lycopersicum L.) was undertaken to evaluate tomato genotypes for variability in yield and yield attributes and screening the genotypes for tolerance/ resistance to early blight.

\section{MATERIALS AND METHODS}

The present investigation was carried out in the Experimental Farm, Department of Horticulture, Assam Agricultural University, Jorhat. Forty-five (Table-1) tomato genotypes were planted during Rabi season 2012-13 and 2013-14 consecutively in a randomized block design with two replications. The crops were grown following recommended package of practices. They were evaluated for yield and resistance to early blight in field conditions.

Observations were taken from five randomly selected plants from each treatment and each replication in the field trial conducted as specified below to screen the cultivars against the pathogen. The disease severity was assessed on all leaves and scored on 0-5 points scale as suggested by Pandey et al. (2003) and percent disease incidence (PDI) was calculated following Mckinney (1923) formula. Later, the disease reaction based on PDI (Fig.3) was recorded according to the scale given by Peteira et al. (2002). After 7 days of incubation, plants were individually evaluated for disease scoring following disease scale (Pandey et al., 2003). The percentage of infection on the leaves were observed and recorded.

The disease severity was scored on a five-point scale:

0 - Free from infection,

1 -One or two necrotic spots on a few lower leaves of plants,

2 - A few isolated spots on leaves, covering nearly $5-10 \%$ of the surface area of the plant,

3 - Many spots coalesced on the leaves, covering 25\% of the surface area of the plant,

4 - Irregular, blighted leaves and sunken lesions with prominent concentric rings on the stem, petiole, and fruit, covering $40-50 \%$ of the surface area,

5 - Whole plant blighted, leaves and fruits starting to fall; foliar part free of disease.

From the disease scored obtained from the above five point scale, percent disease index (PDI) (McKinney, 1923; Pandey et al., 2003) is calculated.

Sum of all rating X 100

$$
\text { PDI }=\frac{\text { Total no. of observations X maximum }}{\text { Tating grade }}
$$$$
\text { rating grade }
$$

After finding out the PDI values, the disease reaction classes for early blight infection based on percent disease severity in tomato were given as prepared by Peteira et al. (2002).

\section{Disease reaction \\ Highly resistant PDI range \\ Resistant \\ Moderately resistant \\ Susceptible \\ $0-12.5$ \\ $12.6-25.0$ \\ $25.1-37.5$ \\ $37.6-50.0$ \\ Highly susceptible $\quad 50.1$ and above}

The yield per ha data from each genotype obtained from disease free condition and that from disease infested conditions were taken separately to find out the loss in yield due to disease incidence. Later, it is calculated in percentage loss.

\section{RESULTS AND DISCUSSION}

The tomato genotypes differed in their resistance reaction against early blight (Table -3). Of the total forty five genotypes screened, Sel-35 (TLBRH-6 X Konbilahi) and Sel-19 (TLBRH-6 X Konbilahi) were highly resistant (which are the selections in segregating generations of the crosses between TLBRH-6 and $S$. pimpinellifolium L. [formerly L. pimpinellifolium (L.) Mill.] with PDI value range (0-12.5\%), 7 varieties were resistant with PDI value range (12.6-12.5\%), 14 were moderately resistant with PDI value range (25.6 $-37.5 \%), 16$ were susceptible with PDI value range $(37.6-50 \%)$ and 6 were highly susceptible with PDI value range ( 50.1 and above $\%$ ) under field condition after inoculation during both years (Tables 2-4, Fig. 1). The genotype with earliness, high yield (Khaidem et al., 2014) and resistance to early blight was 10 / 
Table 1. Genotypes used for study and their salient characteristics.

\begin{tabular}{|c|c|c|c|c|c|c|}
\hline $\begin{array}{l}\text { S. } \\
\text { N. }\end{array}$ & Genotypes & Source & Plant type & $\begin{array}{l}\text { Duration } \\
\text { (days) }\end{array}$ & Fruit size & $\begin{array}{l}\text { Fruit } \\
\text { shape }\end{array}$ \\
\hline 1 & 2012/TOLCVRES-1 & AICRP (VC) & Determinate & 124 & Medium & Oval \\
\hline 2 & 2012/TOLCVRES-2 & AICRP (VC) & Determinate & 122 & Medium & Flat \\
\hline 3 & 2012/TOLCVRES-3 & AICRP (VC) & Determinate & 111 & Large & Oblong \\
\hline 4 & 2012/TOLCVRES-4 & AICRP (VC) & Determinate & 122 & Medium & Round \\
\hline 5 & 2012/TOLCVRES-5 & AICRP (VC) & Determinate & 119 & Medium & Round \\
\hline 6 & 2012/TOLCVRES-6 & AICRP (VC) & Determinate & 124 & Medium & Round \\
\hline 7 & 2012/TOLCVRES-7 & AICRP (VC) & Determinate & 120 & Large & Round \\
\hline 8 & 2012/TOLCVRES-8 & AICRP (VC) & Determinate & 124 & Large & Round \\
\hline 9 & 2012/TOLCVRES-9 & AICRP (VC) & Determinate & 117 & Medium & Round \\
\hline 10 & 2012/SPT/TOINDVAR-1 & AICRP (VC) & Indeterminate & 131 & Medium & Round \\
\hline 11 & 2012/SPT/TOINDVAR-2 & AICRP (VC) & Indeterminate & 133 & Large & Oblong \\
\hline 12 & 2012/SPT/TOINDVAR-3 & AICRP (VC) & Indeterminate & 131 & Medium & Oval \\
\hline 13 & 2012/SPT/TOINDVAR-4 & AICRP (VC) & Indeterminate & 130 & Medium & Round \\
\hline 14 & 2012/SPT/TOINDVAR-5 & AICRP (VC) & Indeterminate & 130 & Medium & Round \\
\hline 15 & 2012/SPT/TOINDVAR-6 & AICRP (VC) & Indeterminate & 122 & Medium & Round \\
\hline 16 & 2012/SPT/TOINDVAR-7 & AICRP (VC) & Indeterminate & 132 & Medium & Flat round \\
\hline 17 & 2012/SPT/TOINDVAR-8 & AICRP (VC) & Indeterminate & 130 & Large & Flat round \\
\hline 18 & 2012/SPT/TOINDVAR-9 & AICRP (VC) & Indeterminate & 134 & Medium & Round \\
\hline 19 & 2012/SPT/TOINDVAR-10 & AICRP (VC) & Indeterminate & 124 & Medium & Round \\
\hline 20 & 2012/SPT/TODVAR-1 & AICRP (VC) & Determinate & 117 & Medium & Round \\
\hline 21 & 2012/SPT/TODVAR-2 & AICRP (VC) & Determinate & 117 & Large & Flat round \\
\hline 22 & 2012/SPT/TODVAR-3 & AICRP (VC) & Determinate & 120 & Medium & Round \\
\hline 23 & 2012/SPT/TODVAR-4 & AICRP (VC) & Determinate & 110 & Medium & Round \\
\hline 24 & 2012/SPT/TODVAR-5 & AICRP (VC) & Determinate & 120 & Medium & Oval \\
\hline 25 & 2012/SPT/TODVAR-6 & AICRP (VC) & Determinate & 123 & Medium & Round \\
\hline 26 & 2012/SPT/TODVAR-7 & AICRP (VC) & Determinate & 122 & Medium & Pear \\
\hline 27 & 2012/SPT/TODVAR-8 & AICRP (VC) & Determinate & 123 & Medium & Round \\
\hline 28 & 2012/SPT/TODVAR-9 & AICRP (VC) & Determinate & 121 & Medium & Round \\
\hline 29 & 2012/SPT/TODVAR10 & AICRP (VC) & Determinate & 117 & Medium & Round \\
\hline 30 & 10/TOLCVRES-1 & AICRP (VC) & Determinate & 122 & Medium & Flat round \\
\hline 31 & 10/TOLCVRES-2 & AICRP (VC) & Determinate & 118 & Large & Pear \\
\hline 32 & 10/TOLCVRES-3 & AICRP (VC) & Determinate & 117 & Medium & Oval \\
\hline 33 & 10/TOLCVRES-5 & AICRP (VC) & Determinate & 115 & Medium & Round \\
\hline 34 & 10/TOLCVRES-6 & AICRP (VC) & Determinate & 118 & Medium & Round \\
\hline 35 & $\begin{array}{l}\text { Sel-35(TLBRH-6 X Kon- } \\
\text { bilahi) }\end{array}$ & AAU, Jorhat & Indeterminate & 130 & Small & Round \\
\hline 36 & $\begin{array}{l}\text { Sel-19 (TLBRH-6 X Kon- } \\
\text { bilahi) }\end{array}$ & AAU, Jorhat & Indeterminate & 128 & Small & Round \\
\hline 37 & Sel-46 (H-24 X Konbilahi) & AAU, Jorhat & $\begin{array}{l}\text { Semi- } \\
\text { indeterminate }\end{array}$ & 125 & Small & Round \\
\hline 38 & Sel-16 (H-24 X Konbilahi) & AAU, Jorhat & Indeterminate & 122 & Small & Round \\
\hline 39 & $\begin{array}{l}\text { Sel-9 (TLBRH-5 X Konbi- } \\
\text { lahi) }\end{array}$ & AAU, Jorhat & $\begin{array}{l}\text { Semi- } \\
\text { indeterminate }\end{array}$ & 126 & Small & Round \\
\hline 40 & Arka vikas & $\begin{array}{l}\text { IIHR, Banga- } \\
\text { lore }\end{array}$ & $\begin{array}{l}\text { Semi- Determi- } \\
\text { nate }\end{array}$ & 124 & Medium & Oval \\
\hline 41 & Hisar Arun & HAU, Hisar & Determinate & 118 & Medium & Round \\
\hline 42 & $\mathrm{H}-86$ & IIVR,Varanasi & Determinate & 117 & Medium & Flat round \\
\hline 43 & Punjab Chhuhara(C) & $\begin{array}{l}\text { PAU, Ludhi- } \\
\text { ana }\end{array}$ & Determinate & 116 & Medium & Oblong \\
\hline 44 & H-24(C) & $\begin{array}{l}\text { IIVR, Vara- } \\
\text { nasi }\end{array}$ & Determinate & 106 & $\begin{array}{l}\text { Slightly } \\
\text { small }\end{array}$ & Round \\
\hline 45 & NDT-3(C) & $\begin{array}{l}\text { NDUAT, Fai- } \\
\text { zabad }\end{array}$ & $\begin{array}{l}\text { Semi- } \\
\text { indeterminate }\end{array}$ & 128 & Medium & Oval \\
\hline
\end{tabular}

Note: AICRP (VC)-All India Coordinated Research Project (Vegetable Crops), AAU-Assam Agricultural University, IIHRIndian Institute of Horticultural research, HAU-Haryana Agricultural University, IIVR-Indian Institute of Vegetable Research, NDUAT- Narendra Deva University of Agriculture \& Technology 
Table 2. Percent disease incidence of early blight in the tomato genotypes.

\begin{tabular}{|c|c|c|c|c|c|c|c|c|}
\hline \multirow{3}{*}{$\begin{array}{c}\text { Genotypes/Sources } \\
\text { of Origin }\end{array}$} & \multicolumn{6}{|c|}{ Percent disease index (PDI) } & \multirow{2}{*}{\multicolumn{2}{|c|}{$\begin{array}{c}\text { Score } \\
37 \text { Days after in- } \\
\text { oculation }\end{array}$}} \\
\hline & \multicolumn{2}{|c|}{$\begin{array}{c}7 \text { Days after inocu- } \\
\text { lation }\end{array}$} & \multicolumn{2}{|c|}{$\begin{array}{c}\text { 22Days after in- } \\
\text { oculation }\end{array}$} & \multicolumn{2}{|c|}{$\begin{array}{c}37 \text { Days after in- } \\
\text { oculation }\end{array}$} & & \\
\hline & $2012-13$ & 2013-14 & 2012-13 & 2013-14 & 2012-13 & 2013-14 & 2012-13 & 2013-14 \\
\hline 2012/TOLCVRES-1/ & $\begin{array}{c}39.25 \\
(38.78)^{\mathrm{a}}\end{array}$ & $\begin{array}{c}42.34 \\
(40.58)^{\mathrm{a}}\end{array}$ & $\begin{array}{c}51.08 \\
(45.60)^{\mathrm{bc}}\end{array}$ & $\begin{array}{c}54.45 \\
(47.56)^{\mathrm{a}}\end{array}$ & $\begin{array}{c}73.56 \\
(59.04)^{\mathrm{b}}\end{array}$ & $\begin{array}{c}72.25 \\
(58.19)^{\mathrm{b}}\end{array}$ & 5 & 5 \\
\hline 2012/TOLCVRES-2/ & $\begin{array}{c}27.04 \\
(31.30)^{\text {defg }}\end{array}$ & $\begin{array}{c}27.45 \\
\left(\begin{array}{c}(31.56) \\
\text { sdef }\end{array}\right.\end{array}$ & $\begin{array}{c}36.20 \\
(36.97)^{\text {fgh }}\end{array}$ & $\begin{array}{c}37.35 \\
(37.62)^{\mathrm{e}}\end{array}$ & $\begin{array}{c}48.32 \\
(44.02)^{\text {ef }}\end{array}$ & $\begin{array}{c}49.33 \\
(44.60)^{\text {ef }}\end{array}$ & 4 & 4 \\
\hline 2012/TOLCVRES-3/ & $\begin{array}{c}11.98 \\
(20.19)^{\mathrm{qr}}\end{array}$ & $\begin{array}{c}10.24 \\
(18.59)^{\mathrm{st}}\end{array}$ & $\begin{array}{c}12.66 \\
(20.83)^{\mathrm{st}}\end{array}$ & $\begin{array}{c}14.35 \\
(22.35)^{\mathrm{tv}}\end{array}$ & $\begin{array}{c}14.34 \\
(22.24)^{\mathrm{uv}}\end{array}$ & $\begin{array}{c}20.34 \\
(26.80)^{\mathrm{r}}\end{array}$ & 2 & 2 \\
\hline 2012/TOLCVRES-4/ & $\begin{array}{c}21.53 \\
(27.63)^{\mathrm{ijkl}}\end{array}$ & $\begin{array}{l}20.12 \\
(26.64) \\
\text { hijklmno }\end{array}$ & $\begin{array}{c}29.28 \\
(32.74) \\
\text { klmn }\end{array}$ & $\begin{array}{c}27.45 \\
(31.65)^{\operatorname{lmn}}\end{array}$ & $\begin{array}{c}35.55 \\
(36.58) \\
\text { Imno }\end{array}$ & $\begin{array}{c}32.12 \\
(34.51) \\
\text { opq }\end{array}$ & 3 & 3 \\
\hline 2012/TOLCVRES-5/ & $\begin{array}{c}41.23 \\
(39.93)^{\mathrm{a}}\end{array}$ & $\begin{array}{c}39.09 \\
(38.68)^{\mathrm{a}}\end{array}$ & $\begin{array}{c}55.33 \\
(48.04)^{\mathrm{a}}\end{array}$ & $\begin{array}{c}53.69 \\
(46.60)^{\mathrm{b}}\end{array}$ & $\begin{array}{c}79.43 \\
(63.03)^{\mathrm{a}}\end{array}$ & $\begin{array}{c}77.53 \\
(61.70)^{\mathrm{a}}\end{array}$ & 5 & 5 \\
\hline 2012/TOLCVRES-6/ & $\begin{array}{c}22.35 \\
(28.20)^{\mathrm{hijkl}}\end{array}$ & $\begin{array}{l}23.00 \\
(28.64) \\
\text { efghijk }\end{array}$ & $\begin{array}{c}31.24 \\
(33.97)^{\mathrm{jkl}}\end{array}$ & $\begin{array}{c}29.76 \\
(32.74) \\
\text { jklm }\end{array}$ & $\begin{array}{c}36.44 \\
(37.11) \\
\text { klmno }\end{array}$ & $\begin{array}{c}36.99 \\
(37.44) \\
j \mathrm{klm}\end{array}$ & 3 & 3 \\
\hline 2012/TOLCVRES-7/ & $\begin{array}{c}23.02 \\
(28.65)^{\mathrm{hijk}}\end{array}$ & $\begin{array}{l}21.99 \\
(27.95) \\
\text { efghijkl }\end{array}$ & $\begin{array}{c}33.22 \\
(35.18)^{\mathrm{kij}}\end{array}$ & $\begin{array}{c}35.24 \\
\left(\begin{array}{c}36.43) \\
\text { efgh }\end{array}\right.\end{array}$ & $\begin{array}{c}42.54 \\
(40.69) \\
\text { ghij }\end{array}$ & $\begin{array}{c}41.21 \\
(39.92)^{\mathrm{hij}}\end{array}$ & 4 & 4 \\
\hline 2012/TOLCVRES-8/ & $\begin{array}{c}40.01 \\
(39.22)^{\mathrm{a}}\end{array}$ & $\begin{array}{c}33.45 \\
(35.32)^{\mathrm{b}}\end{array}$ & $\begin{array}{c}53.65 \\
(47.08)^{\mathrm{ab}}\end{array}$ & $\begin{array}{c}42.32 \\
(40.74)^{\mathrm{d}}\end{array}$ & $\begin{array}{c}75.32 \\
(60.19)^{\mathrm{ab}}\end{array}$ & $\begin{array}{c}54.45 \\
(47.54)^{\mathrm{d}}\end{array}$ & 5 & 5 \\
\hline 2012/TOLCVRES-9/ & $\begin{array}{c}20.16 \\
(26.67) \\
\text { jklmnop }\end{array}$ & $\begin{array}{l}19.31 \\
(26.06) \\
\text { hijklmnop }\end{array}$ & $\begin{array}{c}25.03 \\
(30.00)^{p}\end{array}$ & $\begin{array}{c}24.31 \\
(29.40)^{\text {nop }}\end{array}$ & $\begin{array}{c}30.43 \\
(33.46)^{\mathrm{pq}}\end{array}$ & $\begin{array}{c}31.21 \\
(33.94) \\
\text { opq }\end{array}$ & 3 & 3 \\
\hline $\begin{array}{l}\text { 2012/SPT/TOINDVAR } \\
-1 /\end{array}$ & $\begin{array}{l}19.35 \\
(26.08) \\
\text { klmno }\end{array}$ & $\begin{array}{l}18.33 \\
(25.34) \\
\text { jklmnopqr }\end{array}$ & $\begin{array}{c}28.25 \\
(32.09) \\
\text { lmno }\end{array}$ & $\begin{array}{c}27.35 \\
(31.44)^{\operatorname{lmn}}\end{array}$ & $\begin{array}{c}36.64 \\
(37.23) \\
\text { klmno }\end{array}$ & $\begin{array}{c}34.11 \\
(35.72) \\
\text { mnopq }\end{array}$ & 3 & 3 \\
\hline $\begin{array}{l}\text { 2012/SPT/TOINDVAR } \\
-2 /\end{array}$ & $\begin{array}{l}20.25 \\
(26.73) \\
\text { jklmnop }\end{array}$ & $\begin{array}{l}18.73 \\
(25.63) \\
\text { ijklmnopq }\end{array}$ & $\begin{array}{c}29.14 \\
(32.66) \\
\text { klmn }\end{array}$ & $\begin{array}{c}27.35 \\
(31.51)^{\operatorname{lmn}}\end{array}$ & $\begin{array}{l}35.35 \\
(36.46) \\
\text { lmnop }\end{array}$ & $\begin{array}{c}36.47 \\
(37.13) \\
\text { klmn }\end{array}$ & 4 & 4 \\
\hline $\begin{array}{l}\text { 2012/SPT/TOINDVAR } \\
-3 /\end{array}$ & $\begin{array}{c}35.36 \\
(36.47)^{\mathrm{bc}}\end{array}$ & $\begin{array}{c}24.31 \\
\left(\begin{array}{l}29.53) \\
\text { defghij }\end{array}\right.\end{array}$ & $\begin{array}{c}49.71 \\
(44.82)^{\mathrm{c}}\end{array}$ & $\begin{array}{c}36.35 \\
(37.07)^{\text {ef }}\end{array}$ & $\begin{array}{c}72.56 \\
(58.41)^{\mathrm{b}}\end{array}$ & $\begin{array}{c}58.78 \\
(50.04)^{\mathrm{c}}\end{array}$ & 5 & 5 \\
\hline $\begin{array}{l}\text { 2012/SPT/TOINDVAR } \\
-4 /\end{array}$ & $\begin{array}{c}12.47 \\
(20.66)^{\mathrm{qr}}\end{array}$ & $\begin{array}{c}13.45 \\
(21.50) \\
\text { pqrst }\end{array}$ & $\begin{array}{c}17.25 \\
(24.52)^{\mathrm{qr}}\end{array}$ & $\begin{array}{c}16.34 \\
(24.05)^{\mathrm{st}}\end{array}$ & $\begin{array}{c}24.34 \\
(29.55)^{\mathrm{rs}}\end{array}$ & $\begin{array}{l}20.24 \\
(26.72)^{r}\end{array}$ & 2 & 2 \\
\hline $\begin{array}{l}\text { 2012/SPT/TOINDVAR } \\
-5 /\end{array}$ & $\begin{array}{c}28.35 \\
(32.16)^{\mathrm{de}}\end{array}$ & $\begin{array}{c}25.33 \\
(30.20) \\
\text { defgh }\end{array}$ & $\begin{array}{c}39.60 \\
(38.98)^{\mathrm{de}}\end{array}$ & $\begin{array}{c}35.24 \\
(36.36) \\
\text { efgh }\end{array}$ & $\begin{array}{c}50.00 \\
(44.98)^{\mathrm{e}}\end{array}$ & $\begin{array}{c}42.33 \\
(40.57)^{\mathrm{hi}}\end{array}$ & 5 & 5 \\
\hline $\begin{array}{l}\text { 2012/SPT/TOINDVAR } \\
-6 /\end{array}$ & $\begin{array}{c}27.16 \\
(31.39)^{\mathrm{def}}\end{array}$ & $\begin{array}{l}21.35 \\
(27.50) \\
\text { fghijklm }\end{array}$ & $\begin{array}{c}38.71 \\
(38.46)^{\mathrm{ef}}\end{array}$ & $\begin{array}{c}33.25 \\
(35.18)^{\text {fghi }}\end{array}$ & $\begin{array}{c}49.54 \\
(44.72)^{\mathrm{ef}}\end{array}$ & $\begin{array}{c}40.33 \\
(39.41) \\
\text { hijk }\end{array}$ & 4 & 4 \\
\hline $\begin{array}{l}\text { 2012/SPT/TOINDVAR } \\
-7 /\end{array}$ & $\begin{array}{c}30.22 \\
(33.33)^{d}\end{array}$ & $\begin{array}{c}29.45 \\
(32.85) \\
\text { bcd }\end{array}$ & $\begin{array}{c}41.66 \\
(40.18)^{d}\end{array}$ & $\begin{array}{c}37.55 \\
(37.62)^{\mathrm{e}}\end{array}$ & $\begin{array}{c}49.50 \\
(44.70)^{\mathrm{ef}}\end{array}$ & $\begin{array}{c}47.64 \\
(43.63)^{\mathrm{ef}}\end{array}$ & 5 & 5 \\
\hline $\begin{array}{l}\text { 2012/SPT/TOINDVAR } \\
-8 /\end{array}$ & $\begin{array}{c}13.10 \\
(21.20)^{\mathrm{qr}}\end{array}$ & $\begin{array}{c}12.20 \\
(20.42)^{\mathrm{rst}}\end{array}$ & $\begin{array}{c}15.24 \\
(22.96)^{\mathrm{rs}}\end{array}$ & $\begin{array}{c}17.34 \\
(24.92)^{\mathrm{rst}}\end{array}$ & $\begin{array}{c}23.44 \\
(28.94)^{\mathrm{rs}}\end{array}$ & $\begin{array}{c}24.10 \\
(29.38)^{r}\end{array}$ & 3 & 3 \\
\hline $\begin{array}{l}\text { 2012/SPT/TOINDVAR } \\
-9 /\end{array}$ & $\begin{array}{c}34.25 \\
(35.80)^{\mathrm{d}}\end{array}$ & $\begin{array}{c}25.25 \\
(30.15) \\
\text { defghi }\end{array}$ & $\begin{array}{c}42.34 \\
(40.58)^{\mathrm{d}}\end{array}$ & $\begin{array}{c}36.66 \\
(37.15)^{\mathrm{ef}}\end{array}$ & $\begin{array}{c}54.56 \\
(47.60)^{\mathrm{d}}\end{array}$ & $\begin{array}{c}45.44 \\
(42.37)^{\mathrm{fg}}\end{array}$ & 4 & 4 \\
\hline $\begin{array}{l}\text { 2012/SPT/TOINDVAR } \\
-10 /\end{array}$ & $\begin{array}{c}18.90 \\
(25.75)^{\operatorname{lmno}}\end{array}$ & $\begin{array}{l}21.01 \\
(27.26) \\
\text { fghijklm }\end{array}$ & $\begin{array}{c}29.00 \\
(32.57) \\
\text { klmno }\end{array}$ & $\begin{array}{c}27.45 \\
(31.44)^{\operatorname{lmn}}\end{array}$ & $\begin{array}{c}36.46 \\
(37.13) \\
\text { klmno }\end{array}$ & $\begin{array}{c}35.44 \\
(36.52) \\
\text { lmno }\end{array}$ & 3 & 3 \\
\hline 2012/SPT/TODVAR-1/ & $\begin{array}{c}17.34 \\
(24.59)^{\text {nop }}\end{array}$ & $\begin{array}{c}18.33 \\
(25.33) \\
\text { jklmnopqr }\end{array}$ & $\begin{array}{c}26.75 \\
(31.13)^{\text {nop }}\end{array}$ & $\begin{array}{c}23.13 \\
(28.78)^{\mathrm{op}}\end{array}$ & $\begin{array}{c}33.67 \\
(35.45) \\
\text { nopq }\end{array}$ & $\begin{array}{c}32.22 \\
(34.57) \\
\text { nopq }\end{array}$ & 3 & 3 \\
\hline 2012/SPT/TODVAR-2/ & $\begin{array}{c}22.44 \\
(28.26)^{\mathrm{hijkl}}\end{array}$ & $\begin{array}{c}20.73 \\
(27.07) \\
\text { ghijklmn }\end{array}$ & $\begin{array}{l}32.14 \\
(34.52)^{\mathrm{ijk}}\end{array}$ & $\begin{array}{c}31.35 \\
(34.20)^{\mathrm{hijk}}\end{array}$ & $\begin{array}{c}37.50 \\
\left(\begin{array}{c}37.75) \\
\text { klmno }\end{array}\right.\end{array}$ & $\begin{array}{c}37.00 \\
\left(\begin{array}{c}37.44) \\
\text { jklm }\end{array}\right.\end{array}$ & 4 & 4 \\
\hline
\end{tabular}


Contd...

\begin{tabular}{|c|c|c|c|c|c|c|c|c|}
\hline $\begin{array}{l}\text { 2012/SPT/ } \\
\text { TODVAR-3/ }\end{array}$ & $\begin{array}{c}21.45 \\
(27.57)^{\mathrm{ijklm}}\end{array}$ & $\begin{array}{c}21.75 \\
(27.78)^{\text {fghijkl }}\end{array}$ & $\begin{array}{c}35.08 \\
(36.30)^{\text {ghi }}\end{array}$ & $\begin{array}{c}32.66 \\
(34.74)^{\text {ghij }}\end{array}$ & $\begin{array}{c}44.66 \\
(41.92)^{\mathrm{fgh}}\end{array}$ & $\begin{array}{c}42.24 \\
(40.52)^{\mathrm{hi}}\end{array}$ & 4 & 4 \\
\hline $\begin{array}{l}\text { 2012/SPT/ } \\
\text { TODVAR-4/ }\end{array}$ & $\begin{array}{c}23.46 \\
(28.96)^{\text {ghij }}\end{array}$ & $\begin{array}{c}22.44 \\
(28.26) \\
\text { efghijkl }\end{array}$ & $\begin{array}{c}30.14 \\
(33.28)^{\mathrm{klm}}\end{array}$ & $\begin{array}{c}32.33 \\
(34.50)^{\text {ghij }}\end{array}$ & $\begin{array}{c}38.64 \\
(38.42)^{\mathrm{ijk} k \mathrm{mn}}\end{array}$ & $\begin{array}{c}40.44 \\
(39.47)^{\mathrm{hijk}}\end{array}$ & 4 & 4 \\
\hline $\begin{array}{l}\text { 2012/SPT/ } \\
\text { TODVAR-5/ }\end{array}$ & $\begin{array}{c}13.45 \\
(21.50)^{\mathrm{qr}}\end{array}$ & $\begin{array}{c}15.21 \\
(22.94)^{\text {nopqrs }}\end{array}$ & $\begin{array}{c}14.35 \\
(22.24)^{\mathrm{rs}}\end{array}$ & $\begin{array}{c}16.25 \\
(23.65)^{\mathrm{st}}\end{array}$ & $\begin{array}{c}18.65 \\
(25.56)^{\text {tu }}\end{array}$ & $\begin{array}{c}20.14 \\
(26.64)^{r}\end{array}$ & 2 & 2 \\
\hline $\begin{array}{l}\text { 2012/SPT/ } \\
\text { TODVAR-6/ }\end{array}$ & $\begin{array}{c}17.35 \\
(24.60)^{\text {nop }}\end{array}$ & $\begin{array}{c}15.45 \\
(30.45)^{\text {defg }}\end{array}$ & $\begin{array}{c}19.25 \\
(26.01)^{\mathrm{q}}\end{array}$ & $\begin{array}{c}19.85 \\
(26.28)^{\mathrm{qrs}}\end{array}$ & $\begin{array}{c}23.34 \\
(28.87)^{\mathrm{rs}}\end{array}$ & $\begin{array}{c}24.00 \\
(29.32)^{\mathrm{r}}\end{array}$ & 2 & 2 \\
\hline $\begin{array}{l}\text { 2012/SPT/ } \\
\text { TODVAR-7/ }\end{array}$ & $\begin{array}{c}21.34 \\
(27.48)^{\mathrm{ijklm}}\end{array}$ & $\begin{array}{c}20.01 \\
(26.54) \\
\text { hijklmno }\end{array}$ & $\begin{array}{c}26.54 \\
(30.99)^{\text {nop }}\end{array}$ & $\begin{array}{c}24.31 \\
(29.66)^{\text {nop }}\end{array}$ & $\begin{array}{c}30.21 \\
(33.33)^{\mathrm{q}}\end{array}$ & $\begin{array}{c}30.99 \\
(33.81)^{\mathrm{pq}}\end{array}$ & 3 & 3 \\
\hline $\begin{array}{l}\text { 2012/SPT/ } \\
\text { TODVAR-8/ }\end{array}$ & $\begin{array}{c}19.08 \\
(25.88)^{\operatorname{lmno}}\end{array}$ & $\begin{array}{l}19.09 \\
(25.89) \\
\text { hijklmnop }\end{array}$ & $\begin{array}{c}27.33 \\
(31.50) \\
\text { mnop }\end{array}$ & $\begin{array}{c}24.13 \\
(29.65)^{\text {nop }}\end{array}$ & $\begin{array}{c}32.35 \\
(34.65)^{\mathrm{opq}}\end{array}$ & $\begin{array}{c}30.12 \\
(33.27)^{\mathrm{q}}\end{array}$ & 3 & 3 \\
\hline $\begin{array}{l}\text { 2012/SPT/ } \\
\text { TODVAR-9/ }\end{array}$ & $\begin{array}{c}28.21 \\
(32.06)^{\mathrm{de}}\end{array}$ & $\begin{array}{c}33.13 \\
(35.12)^{\mathrm{bc}}\end{array}$ & $\begin{array}{c}37.10 \\
(37.51)^{\mathrm{fg}}\end{array}$ & $\begin{array}{c}42.43 \\
(40.67)^{\mathrm{d}}\end{array}$ & $\begin{array}{c}46.23 \\
(42.82)^{\mathrm{efg}}\end{array}$ & $\begin{array}{c}50.00 \\
(44.98)^{\mathrm{e}}\end{array}$ & 4 & 4 \\
\hline $\begin{array}{l}\text { 2012/SPT/ } \\
\text { TODVAR10/ }\end{array}$ & $\begin{array}{c}38.25 \\
(38.19)^{\mathrm{ab}}\end{array}$ & $\begin{array}{c}39.12 \\
(38.70)^{\mathrm{a}}\end{array}$ & $\begin{array}{c}49.21 \\
(44.53)^{\mathrm{c}}\end{array}$ & $\begin{array}{c}47.27 \\
(43.42)^{\mathrm{c}}\end{array}$ & $\begin{array}{c}64.34 \\
(53.31)^{\mathrm{c}}\end{array}$ & $\begin{array}{c}60.45 \\
(51.01)^{\mathrm{c}}\end{array}$ & 5 & 5 \\
\hline 10/TOLCVRES-1/ & $\begin{array}{c}17.67 \\
(24.83)^{\text {nop }}\end{array}$ & $\begin{array}{l}16.24 \\
(23.74) \\
\text { lmnopqrs }\end{array}$ & $\begin{array}{c}28.00 \\
(31.93) \\
\text { lmnop }\end{array}$ & $\begin{array}{c}26.45 \\
(30.79)^{\mathrm{mno}}\end{array}$ & $\begin{array}{c}32.91 \\
(34.98)^{\text {nopq }}\end{array}$ & $\begin{array}{c}30.44 \\
(33.45)^{\mathrm{q}}\end{array}$ & 3 & 3 \\
\hline 10/TOLCVRES-2/ & $\begin{array}{c}25.80 \\
(30.50)^{\text {defgh }}\end{array}$ & $\begin{array}{c}24.31 \\
(29.51)^{\text {defghij }}\end{array}$ & $\begin{array}{c}33.41 \\
(35.29)^{\mathrm{hij}}\end{array}$ & $\begin{array}{c}35.13 \\
(36.55)^{\mathrm{efg}}\end{array}$ & $\begin{array}{c}41.58 \\
(40.14)^{\text {ghijk }}\end{array}$ & $\begin{array}{c}43.00 \\
(40.96)^{\mathrm{gh}}\end{array}$ & 4 & 4 \\
\hline 10/TOLCVRES-3/ & $\begin{array}{c}24.35 \\
(29.55)^{\text {fghi }}\end{array}$ & $\begin{array}{c}23.12 \\
(28.72) \\
\text { defghijk }\end{array}$ & $\begin{array}{c}34.12 \\
(35.73)^{\text {ghij }}\end{array}$ & $\begin{array}{c}30.24 \\
(33.51)^{\mathrm{ijkl}}\end{array}$ & $\begin{array}{c}45.66 \\
(42.49)^{\text {efg }}\end{array}$ & $\begin{array}{c}43.56 \\
(41.28)^{\mathrm{gh}}\end{array}$ & 4 & 4 \\
\hline 10/TOLCVRES-5/ & $\begin{array}{c}21.00 \\
(27.26)^{\mathrm{ijkl} \operatorname{lmn}}\end{array}$ & $\begin{array}{l}19.13 \\
(25.92) \\
\text { hijklmnop }\end{array}$ & $\begin{array}{c}28.00 \\
(31.93) \\
\text { Imnop }\end{array}$ & $\begin{array}{c}27.54 \\
(31.44)^{\operatorname{lmn}}\end{array}$ & $\begin{array}{c}37.68 \\
(37.85)^{\mathrm{jklmn}}\end{array}$ & $\begin{array}{c}37.68 \\
(37.85)^{\mathrm{jklm}}\end{array}$ & 3 & 3 \\
\hline 10/TOLCVRES-6/ & $\begin{array}{c}22.54 \\
(28.33)^{\mathrm{hijkl}}\end{array}$ & $\begin{array}{l}21.15 \\
(27.37) \\
\text { fghijklm }\end{array}$ & $\begin{array}{c}28.71 \\
(32.38) \\
\text { Imno }\end{array}$ & $\begin{array}{c}27.75 \\
(31.44)^{\operatorname{lmn}}\end{array}$ & $\begin{array}{c}36.66 \\
(37.25)^{\mathrm{klmno}}\end{array}$ & $\begin{array}{c}37.00 \\
(37.45)^{\mathrm{jklm}}\end{array}$ & 3 & 3 \\
\hline Sel-35/ & $\begin{array}{c}10.23 \\
(18.64)^{r}\end{array}$ & $\begin{array}{c}7.75 \\
(16.15)^{t}\end{array}$ & $\begin{array}{c}12.25 \\
(20.47)^{\mathrm{st}}\end{array}$ & $\begin{array}{c}11.46 \\
(19.78)^{\mathrm{uv}}\end{array}$ & $\begin{array}{c}12.50 \\
(20.69)^{\mathrm{v}}\end{array}$ & $\begin{array}{c}12.30 \\
(20.51)^{\mathrm{s}}\end{array}$ & 2 & 2 \\
\hline Sel-19/ & $\begin{array}{c}10.50 \\
(18.84)^{\mathrm{r}}\end{array}$ & $\begin{array}{c}7.56 \\
(15.86)^{t}\end{array}$ & $\begin{array}{c}11.20 \\
(19.53)^{\mathrm{t}}\end{array}$ & $\begin{array}{c}10.00 \\
(18.34)^{\mathrm{v}}\end{array}$ & $\begin{array}{c}12.00 \\
(20.15)^{\mathrm{v}}\end{array}$ & $\begin{array}{c}11.68 \\
(19.95)^{\mathrm{s}}\end{array}$ & 2 & 2 \\
\hline Sel-46/ & $\begin{array}{c}9.78 \\
(18.20)^{r}\end{array}$ & $\begin{array}{c}12.45 \\
(20.64)^{\mathrm{qrst}}\end{array}$ & $\begin{array}{c}15.23 \\
(22.94)^{\mathrm{rs}}\end{array}$ & $\begin{array}{c}21.70 \\
(27.26)^{\mathrm{pqr}}\end{array}$ & $\begin{array}{c}20.19 \\
(26.68)^{\text {st }}\end{array}$ & $\begin{array}{c}24.34 \\
(29.54)^{\mathrm{r}}\end{array}$ & 2 & 2 \\
\hline Sel-16/ & $\begin{array}{c}15.20 \\
(22.93)^{\mathrm{pq}}\end{array}$ & $\begin{array}{c}14.32 \\
(22.22)^{\text {nopqrs }}\end{array}$ & $\begin{array}{c}17.34 \\
(24.60)^{\mathrm{qr}}\end{array}$ & $\begin{array}{c}19.74 \\
(26.05)^{\mathrm{qrs}}\end{array}$ & $\begin{array}{c}25.34 \\
(30.21)^{\mathrm{r}}\end{array}$ & $\begin{array}{c}24.45 \\
(29.62)^{r}\end{array}$ & 2 & 2 \\
\hline Sel-9/ & $\begin{array}{c}10.28 \\
(18.68)^{r}\end{array}$ & $\begin{array}{c}10.25 \\
(18.65)^{\mathrm{st}}\end{array}$ & $\begin{array}{c}12.13 \\
(20.36)^{\mathrm{st}}\end{array}$ & $\begin{array}{c}11.45 \\
(19.56)^{\mathrm{uv}}\end{array}$ & $\begin{array}{c}13.00 \\
(21.11)^{\mathrm{v}}\end{array}$ & $\begin{array}{c}13.00 \\
(21.11)^{\mathrm{s}}\end{array}$ & 2 & 2 \\
\hline Arka vikas/ & $\begin{array}{c}29.73 \\
(33.03)^{\mathrm{d}}\end{array}$ & $\begin{array}{c}28.42 \\
(32.20)^{\mathrm{bcde}}\end{array}$ & $\begin{array}{c}30.08 \\
(33.25)^{\mathrm{klm}}\end{array}$ & $\begin{array}{c}32.95 \\
(34.50)^{\text {ghij }}\end{array}$ & $\begin{array}{c}38.12 \\
(38.11)^{\mathrm{jklmn}}\end{array}$ & $\begin{array}{c}40.99 \\
(39.79)^{\mathrm{hijk}}\end{array}$ & 4 & 4 \\
\hline $\begin{array}{l}\text { Hisar Arun /(CCS } \\
\text { Haryana Agri. Uni., } \\
\text { Hisar) }\end{array}$ & $\begin{array}{c}21.44 \\
(27.57)^{\mathrm{ijklm}}\end{array}$ & $\begin{array}{c}14.05 \\
(21.99)^{\text {opqrs }}\end{array}$ & $\begin{array}{c}25.77 \\
(30.48)^{\text {op }}\end{array}$ & $\begin{array}{c}22.02 \\
(27.96)^{\mathrm{pq}}\end{array}$ & $\begin{array}{c}33.00 \\
(35.05)^{\text {nopq }}\end{array}$ & $\begin{array}{c}35.24 \\
(36.40)^{\operatorname{lmnop}}\end{array}$ & 3 & 3 \\
\hline H-86/ & $\begin{array}{c}28.29 \\
(32.12)^{\mathrm{de}}\end{array}$ & $\begin{array}{l}17.72 \\
(24.87) \\
\text { kmnopqr }\end{array}$ & $\begin{array}{c}34.48 \\
(35.94)^{\text {ghi }}\end{array}$ & $\begin{array}{c}22.45 \\
(27.93)^{\mathrm{pq}}\end{array}$ & $\begin{array}{c}43.24 \\
(41.10)^{\text {ghi }}\end{array}$ & $\begin{array}{c}38.54 \\
(38.36)^{\mathrm{ijklm}}\end{array}$ & 4 & 4 \\
\hline $\begin{array}{l}\text { Punjab Chhuhara/ } \\
\text { (Punjab Agri. Univ., } \\
\text { Ludhiana) }\end{array}$ & $\begin{array}{c}25.34 \\
(30.20)^{\mathrm{efgh}}\end{array}$ & $\begin{array}{c}24.34 \\
(29.54)^{\text {defghij }}\end{array}$ & $\begin{array}{c}29.12 \\
(32.64) \\
\text { klmn }\end{array}$ & $\begin{array}{c}28.75 \\
(32.15)^{\mathrm{klm}}\end{array}$ & $\begin{array}{c}37.00 \\
(37.44)^{\mathrm{klmno}}\end{array}$ & $\begin{array}{c}37.45 \\
(37.70)^{\mathrm{jklm}}\end{array}$ & 3 & 3 \\
\hline H-24/ & $\begin{array}{c}16.89 \\
(24.25)^{\mathrm{op}}\end{array}$ & $\begin{array}{c}24.75 \\
(29.82)^{\text {defghij }}\end{array}$ & $\begin{array}{c}29.45 \\
(32.84) \\
\text { klmn }\end{array}$ & $\begin{array}{c}32.45 \\
(34.81)^{\text {ghij }}\end{array}$ & $\begin{array}{c}40.35 \\
(39.42)^{\mathrm{hijkl}}\end{array}$ & $\begin{array}{c}39.46 \\
(38.90)^{\mathrm{hijkl}}\end{array}$ & 3 & 3 \\
\hline NDT-3/ & $\begin{array}{c}28.37 \\
(32.17)^{\mathrm{de}}\end{array}$ & $\begin{array}{l}23.37 \\
(28.90) \\
\text { defghijk }\end{array}$ & $\begin{array}{c}36.00 \\
(36.85)^{\text {gh }}\end{array}$ & $\begin{array}{c}36.54 \\
(37.15)^{\mathrm{ef}}\end{array}$ & $\begin{array}{c}45.45 \\
(42.37)^{\mathrm{efg}}\end{array}$ & $\begin{array}{c}42.34 \\
(40.58)^{\mathrm{hi}}\end{array}$ & 4 & 4 \\
\hline C.D. $(5 \%)$ & 3.26 & 5.37 & 2.79 & 3.20 & 4.40 & 3.84 & & \\
\hline S.E. (m) & 1.14 & 1.8 & 0.98 & 1.12 & 1.54 & 1.34 & & \\
\hline
\end{tabular}


TOLCVRES-3 which combined all three desirable characters in tomato. The genotypes with low yield (Table- 4) need to be improved by transferring and accumulating the resistant genes from either Sel-35 or Sel-19 or resistant varieties through backcross breeding or gene pyramiding. Gardner, 1988 developed breeding lines NC63EB, NC870, NCEBR-2, NCEBR-3 and NCEBR-4 from C1943 using as a source of early blight resistance. Upadhyay et al., (2009) also observed that 'EC 520061', wild species of tomato showed appreciable resistance to early blight disease and can be utilized as a source of resistance in future breeding programme. Singh et al., (2011) mentioned about the development of tolerant/resistant tomato plant from wild accessions. Kamble et al. ( 2007) and Mahantesha et al., (2012) reported difference in resistance reaction for tomato plants against early blight disease. It was also evident from the study conducted that there was variations in yield and also reduction in yield due to early blight ranges from $2.15 \%$ (highly resistant, found on genotype Sel-19) to $42.75 \%$ (highly susceptible, found on genotype 2012/ SPT/TOINDVAR-9 ) (Table -4, Fig.2). The loss in yield varied in the current finding and is directly related with the PDI reading. The tomato genotypes that has higher loss but with greater yield advantages may be useful if they are transferred with suitable resistant gene. In susceptible genotypes the loss in yield due to disease incidence ranges from $16.17 \%$ $-19.86 \%$. Similar findings in tomato plant with $78 \%$ loss in yield was also reported by Datar and Mayee ( 1981) and 95\% tomato fruit loss in under severe epiphytic condition as reported by Sridha and Naik (1983).

\section{Conclusion}

The results obtained from the present work have given some important future line of work. The genotypes highly resistant to early blight were Sel-35 and Sel-19 and can be used as parents in hybridization or backcrossing programme in order to transfer the gene for resistance to already adapted varieties or susceptible varieties with desirable characters. The genotypes 2012/SPT/TOINDVAR-4, 2012/SPT/TODVAR-5, 2012/SPT/TODVAR-6, 2012/TOLCVRES-3, Sel-46, Sel-16 and Sel-9 showed resistant against Alternaria solani pathogen which may be further evaluated for stability in performance and for their durable resistance. The genotype 10/TOLCVRES-3 was found good for both high yield and resistant to early blight. Sel-35 and Sel-19 may be studied by combining classical breeding methods with molecular markers in future breeding programme.

\section{REFERENCES}

Barksdale, T.H. and Stoner, A.K. (1977). A study of the inheritance of tomato early blight resistance. Plant Dis. Rep., 61: 63-65.

Balanchard D. (1992). A colour atlas of tomato diseases. 
Table 4. Comparison of yield and its loss percentage between disease free condition and disease infested condition for the year 2012-13.

\begin{tabular}{|c|c|c|c|c|c|c|}
\hline $\begin{array}{l}\text { S. } \\
\text { N. }\end{array}$ & Genotypes & $\begin{array}{l}\text { Resistant } \\
\text { reaction } \\
\text { from PDI } \\
\text { reading }\end{array}$ & $\begin{array}{c}\text { Yield per ha } \\
\text { (q) } \\
\text { (disease free } \\
\text { condition) }\end{array}$ & $\begin{array}{c}\text { Yield per ha } \\
\text { (q) } \\
\text { (disease } \\
\text { infested } \\
\text { condition) }\end{array}$ & $\begin{array}{c}\text { Loss in } \\
\text { yield per } \\
\text { ha }(\mathbf{q}) \\
\text { (due to } \\
\text { disease } \\
\text { incidence) }\end{array}$ & $\begin{array}{c}\% \text { loss in } \\
\text { yield } \\
\text { (due to } \\
\text { disease } \\
\text { incidence) }\end{array}$ \\
\hline 1 & 2012/TOLCVRES-1 & HS & 246.53 & 172.21 & 74.32 & 30.15 \\
\hline 2 & 2012/TOLCVRES-2 & $\mathrm{S}$ & 192.4 & 154.21 & 38.19 & 19.85 \\
\hline 3 & 2012/TOLCVRES-3 & $\mathrm{S}$ & 240.00 & 220.21 & 45.59 & 17.15 \\
\hline 4 & 2012/TOLCVRES-4 & MR & 237.9 & 203.43 & 34.47 & 14.49 \\
\hline 5 & 2012/TOLCVRES-5 & HS & 221.83 & 151.34 & 70.49 & 31.78 \\
\hline 6 & 2012/TOLCVRES-6 & MR & 222.47 & 190.21 & 32.26 & 14.50 \\
\hline 7 & 2012/TOLCVRES-7 & $\mathrm{S}$ & 181.6 & 146.42 & 35.18 & 19.37 \\
\hline 8 & 2012/TOLCVRES-8 & HS & 230.2 & 160.24 & 69.96 & 30.39 \\
\hline 9 & 2012/TOLCVRES-9 & MR & 250.87 & 220.12 & 30.75 & 12.26 \\
\hline 10 & 2012/SPT/TOINDVAR-1 & MR & 205.48 & 176.48 & 29.00 & 14.11 \\
\hline 11 & 2012/SPT/TOINDVAR-2 & $\mathrm{S}$ & 173.43 & 136.42 & 37.01 & 21.34 \\
\hline 12 & 2012/SPT/TOINDVAR-3 & HS & 196.96 & 131.00 & 65.96 & 33.49 \\
\hline 13 & 2012/SPT/TOINDVAR-4 & $\mathrm{R}$ & 126.1 & 118.80 & 7.30 & 5.79 \\
\hline 14 & 2012/SPT/TOINDVAR-5 & $\mathrm{S}$ & 116.55 & 94.42 & 22.13 & 18.99 \\
\hline 15 & 2012/SPT/TOINDVAR-6 & $\mathrm{S}$ & 159.55 & 132.21 & 27.34 & 17.14 \\
\hline 16 & 2012/SPT/TOINDVAR-7 & $\mathrm{S}$ & 145.93 & 116.98 & 28.95 & 19.84 \\
\hline 17 & 2012/SPT/TOINDVAR-8 & MR & 190.69 & 166.59 & 24.10 & 12.64 \\
\hline 18 & 2012/SPT/TOINDVAR-9 & HS & 192.78 & 110.37 & 82.41 & 42.75 \\
\hline 19 & 2012/SPT/TOINDVAR-10 & MR & 211.9 & 186.21 & 25.69 & 12.12 \\
\hline 20 & 2012/SPT/TODVAR-1 & MR & 224.22 & 198.42 & 25.80 & 11.51 \\
\hline 21 & 2012/SPT/TODVAR-2 & MR & 240.13 & 214.24 & 25.89 & 10.78 \\
\hline 22 & 2012/SPT/TODVAR-3 & $\mathrm{S}$ & 264.67 & 212.11 & 52.56 & 19.86 \\
\hline 23 & 2012/SPT/TODVAR-4 & $\mathrm{S}$ & 168.15 & 135.00 & 33.15 & 19.71 \\
\hline 24 & 2012/SPT/TODVAR-5 & $\mathrm{R}$ & 188.52 & 170.21 & 18.31 & 9.71 \\
\hline 25 & 2012/SPT/TODVAR-6 & $\mathrm{R}$ & 244.2 & 230.12 & 14.08 & 5.77 \\
\hline 26 & 2012/SPT/TODVAR-7 & MR & 211.2 & 184.21 & 26.99 & 12.78 \\
\hline 27 & 2012/SPT/TODVAR-8 & MR & 195.68 & 172.42 & 23.26 & 11.89 \\
\hline 28 & 2012/SPT/TODVAR-9 & $\mathrm{S}$ & 235.62 & 189.00 & 46.62 & 19.79 \\
\hline 29 & 2012/SPT/TODVAR10 & HS & 194.7 & 121.72 & 72.98 & 37.48 \\
\hline 30 & 10/TOLCVRES-1 & MR & 201.29 & 178.34 & 22.95 & 11.40 \\
\hline 31 & 10/TOLCVRES-2 & $\mathrm{S}$ & 208.67 & 168.21 & 40.46 & 19.39 \\
\hline 32 & 10/TOLCVRES-3 & $\mathrm{R}$ & 268.82 & 250.12 & 18.70 & 6.96 \\
\hline 33 & 10/TOLCVRES-5 & $\mathrm{S}$ & 196.78 & 158.21 & 38.57 & 19.60 \\
\hline 34 & 10/TOLCVRES-6 & MR & 142.74 & 123.35 & 19.39 & 13.58 \\
\hline 35 & Sel-35 & HR & 80 & 77.56 & 2.44 & 3.05 \\
\hline 36 & Sel-19 & HR & 77.67 & 76.00 & 1.67 & 2.15 \\
\hline 37 & Sel-46 & $\mathrm{R}$ & 95.67 & 86.24 & 9.43 & 9.86 \\
\hline 38 & Sel-16 & $\mathrm{R}$ & 90.67 & 83.46 & 7.21 & 7.95 \\
\hline 39 & Sel-9 & $\mathrm{R}$ & 81.33 & 74.56 & 6.77 & 8.32 \\
\hline 40 & Arka Vikas & $\mathrm{S}$ & 194.48 & 156.22 & 38.26 & 19.67 \\
\hline 41 & Hisar Arun & MR & 205.56 & 182.24 & 23.32 & 11.34 \\
\hline 42 & H-86 & $\mathrm{S}$ & 146.27 & 118.21 & 28.06 & 19.18 \\
\hline 43 & Punjab Chhuhara & MR & 221.76 & 198.24 & 23.52 & 10.61 \\
\hline 44 & $\mathrm{H}-24$ & $\mathrm{~S}$ & 303 & 252.21 & 50.79 & 16.76 \\
\hline 45 & NDT-3 & $\mathrm{S}$ & 258.19 & 212.12 & 46.07 & 17.84 \\
\hline
\end{tabular}




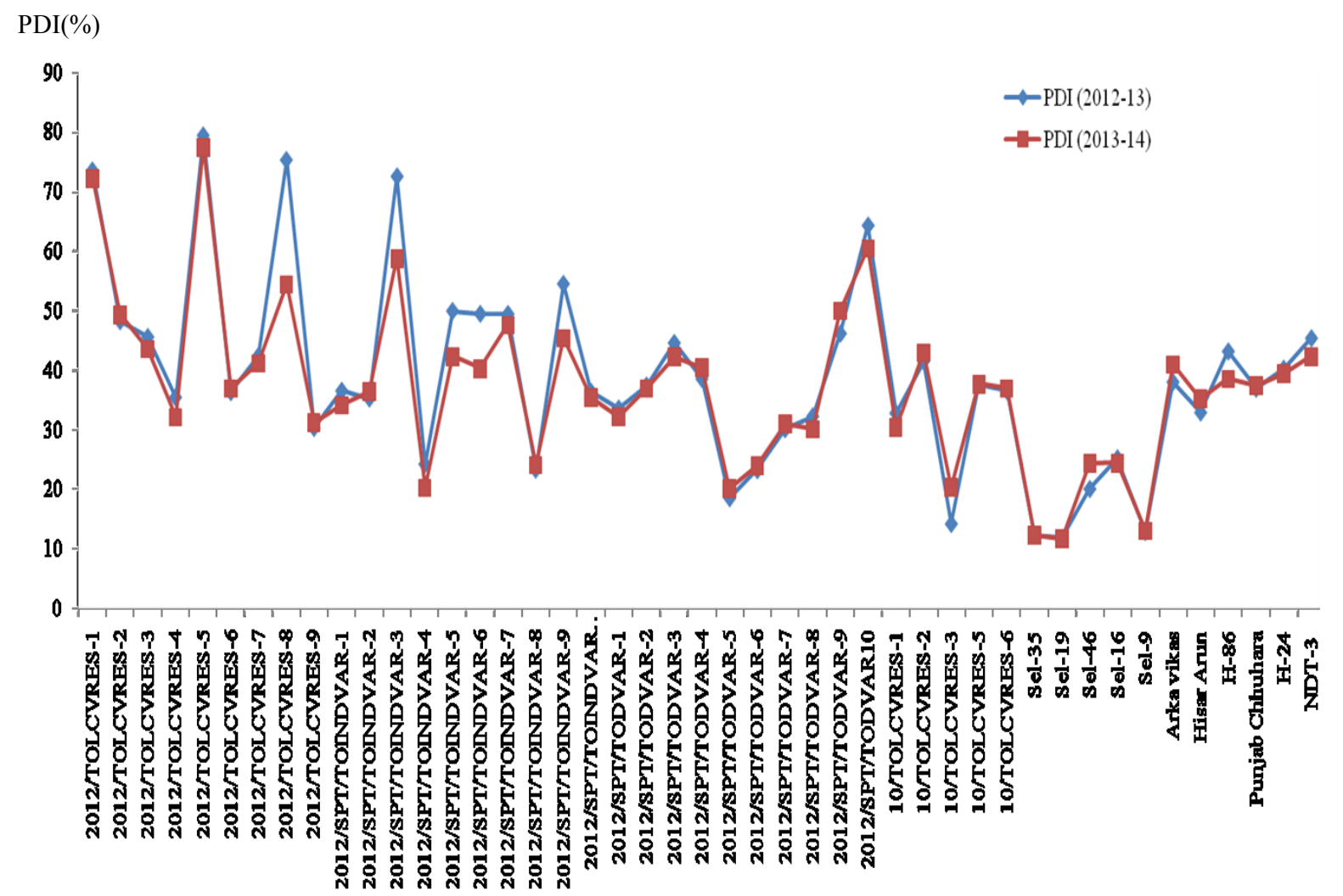

Fig. 1. Comparison of percent disease index (PDI) on the genotypes artificially inoculated by A. solani for both the years 2012 -13 and 2013-14.

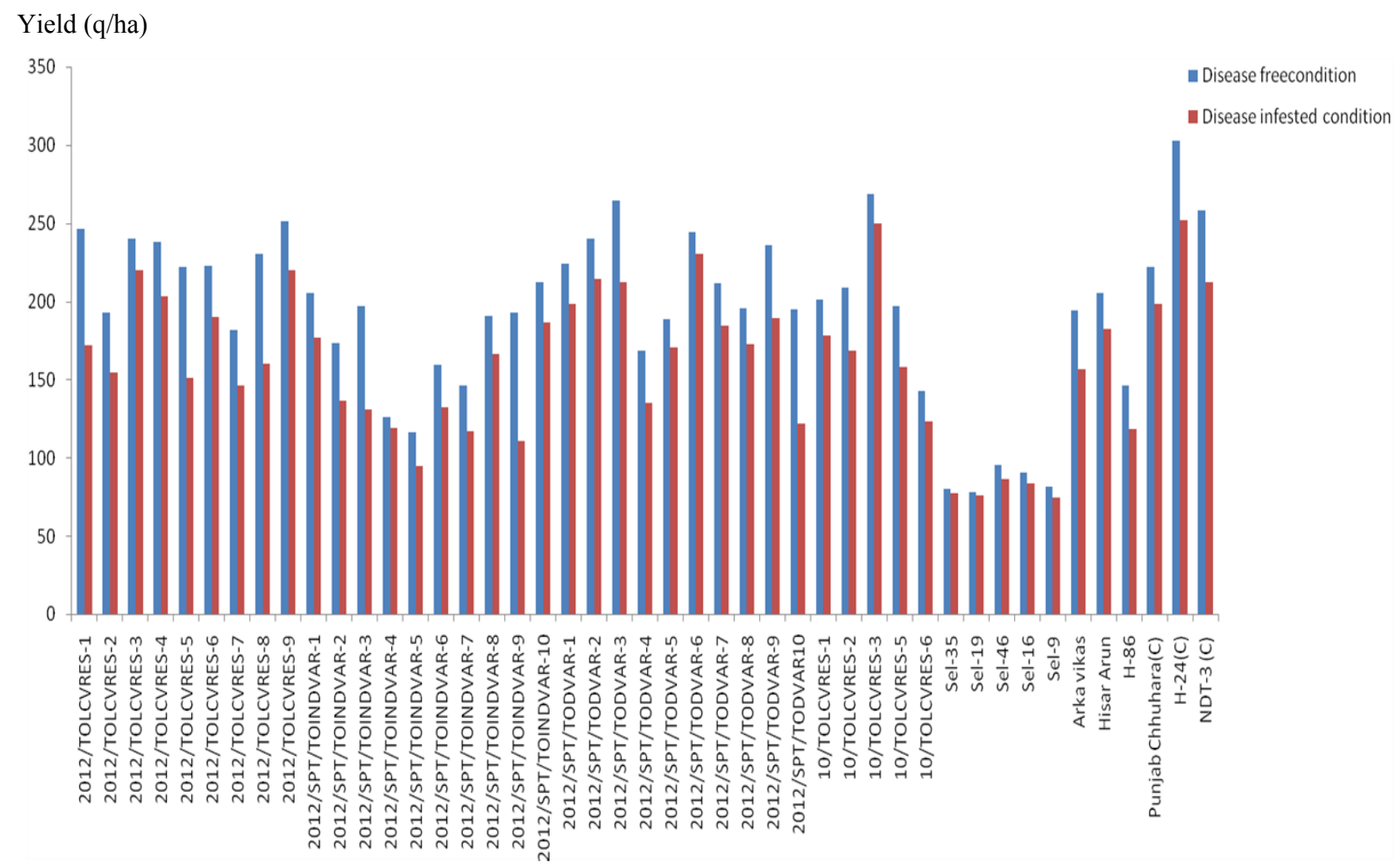

Fig. 2. Yield comparision under early blight free and early blight infested condition during 2012-13. 
Wolfe Pub. Ltd., Brook House, London, pp. 298.

Basu, P.K. (1974). Measuring early blight, its progress and influence on fruit losses in nine tomato cultivars. Canadian Pl. Dis. Survey, 54: 45-50.

Chaerani, R., Groenwold, R., Roel, P.S. and Voorrips, E. (2007). Assessment of early blight (Alternaria solani) resistance in tomato using a droplet inoculation method. J. Gen. Plant Pathol., 73:96-103.

Datar, V.V. and Mayee, C.D. (1981). Assessment of loss in tomato yield due to early blight. Ind. Phytopathology, 34: 191-195.

Fry, W. (2008). Phytophthora infestans: The Plant (and R Gene) Destroyer. Molecular Plant Pathology, 9: 385-340.

Foolad, M.R., Ntahimpera, N., Christ, B.J., and Lin, G.Y. (2000). Comparision of field, green-house and detached leaflet evaluations of tomato germplasm for early blight resistance. Plant Disease, 84: 967-972.

Foolad, M.R., Zhang, L.P., Khan, A.A., Nĩno-Liu, D., and Lin, G.Y. (2002). Identification of QTLs for early blight (Alternaria solani) resistance in tomato using backcross populations of a Lycopersicon esculentum and $L$. hirsutum cross. Theoretical and Applied Genetics, 104 (6-7): 945-958.

Foolad, M.R., Sharma, A., Ashrafi, H. and Lin, G.Y. (2005). Genetics of early blight resistance in tomato. Acta Hort., 695: 397-406.

Gardner, R.G. (1988). NC EBR-1 and NC EBR-2 early blight resistant tomato breeding lines. Hort. Sci. 23: 779 -781 .

Jain, S.K., Kumar, V. and Saharia, M. (2012). Analysis of rainfall and temperature trends in northeast India. Int. J. Climatol, Published online in Wiley Online Library (wileyonlinelibrary.com) DOI: 10.1002/joc.3483.

Kamble, S.B.; Pawar, D.R.; Sankeshwari, S.B.; Parab, M.M. and Arekar, J.S. (2007). Field screening of different tomato varieties and advanced lines against early blight of tomato. Agric. Sci. Digest. 27(4): 267 - 269.

Kumar, S. and Srivastava, K. (2013). Screening of tomato genotypes against early blight (Alternaria solani) under field condition. The Bioscan, 8(1): 189-193.

Kalloo, G. and Banerjee, M.K. (1993). Early blight resistance in Lycopersicon esculentum transferred from $L$. pimpinellifolium and L. hirsutum f. glabratum. Garten bauuissen schaft, 58: 238-39.

Khaidem, M.M., Bora, G.C., Singh, S.J. and Sinha, A.K. (2014). Morphology Based Genetic Variability Analysis and Identification of Important Characters for Tomato (Solanum lycopersicum L.) Crop Improvement. American-Eurasian J. Agric. \& Environ. Sci., 14 (10): 1105-1111.

Mckinney, H.H. (1923). Influence of soil temperature and moisture on infection of wheat seedling by Helminthosporium sativum. J. of Agri. Res., 26: 195-217.

Mahantesha, S.R.; Rawal, R.D. and Ramaswamy, G.R.
(2012). Field evaluation of tomato genotypes for resistance to Alternaria solani. Trends Biosci. 5(4): 294.

Peralta, I.E., Knapp, S. and Spooner, D.M. (2005). Thirteen new species of wild tomatoes (Solanum section Lycopersicon: Solanaceae) from Northern Peru. Syst. Bot., 30: 424-434.

Pandey, K.K., Pandey, P.K., Kalloo, G. and Banerjee, M.K. (2003). Resistance to early blight of tomato with respect to various parameters of disease epidemics. J. Gen. Plant Pathology, 69: 364-371.

Peteira, B., Diaz, M.G. Chavez, Martinez B. and Miranda, I. (2002). Search of a RAPD marker associated to Alternaria solani resistance in tomato. Rev Protection Veg., 17(1): 6-13.

Poysa, V. and Tu, J. (1997). Response of cultivars and breeding lines of Lycopersicon spp. to Alternaria solani. Canadian Plant Disease Survey 76(1): 5- 8.

Rotem, J. and Reichert, I. (1964). Dew - a principal moisture factor enabling early blight epidemics in a semiarid region of Israel. Plant Dis Rep., 48:211-215.

Sharma, A., Zhang, L., Nino-Liu, D., Ashrafi, H. and Foolad, M.R. (2008). A Solanum lycopersicum $\times$ Solanum pimpinellifolium linkage-map of tomato displaying genomic locations of R-genes, RGAs, and candidate resistance/defense-response ESTs. Int. J. Plant Genomics, Article ID 926090, doi:10.1155/2008/926090.

Singh, P.C., Singh, S., Kumar, R., Singh, M. and Rai, M. (2013). Genetic study of early blight resistance in tomato. Indian Society of Vegetable Science, National Symposium on Abiotic and Biotic Stress Management in Vegetable Crops.

Singh, P.C., Kumar, R., Singh, M., Rai, A., Singh, M.C., and Rai, M. (2011). Identification of resistant sources against early blight disease of tomato. Indian J. Hort., 68(4): 516-521.

Sherf, A.F., MacNab, A.A. (1986) Vegetable diseases and their control. Wiley, New York.

Singh, R.S. (1985). Disease of vegetable crops. Oxford and IBH Publishing Co. New Delhi, pp. 441.

Sridha, T.S. and Naik, L.B. (1983). Relative resistance of tomato cultivars to early blight. The Madras Agric. J., 70: 488-489.

Upadhyay, P., Singh, P.C., Sinha, B., Singh, M., Kumar, R., Pandey, K. K. and Rai, M. (2009). Sources of resistance against early blight (Alternaria solani) in tomato (Solanum lycopersicum). Ind. J. Agric. Sci. 79(9): 752-753.

Thirthammallappa and Lohithaswa, H.C. (2000). Genetics of resistance to early blight (Alternaria solani) in tomato. Euphytica, 113: 187-197.

Yadav, D.S. and Singh, S.P. (1998). Correlation and path analysis in tomato. J. of Hill Res., 112(2): 207-211.

Yadav, O.P. and Dabbas, M.R. (2012). Efficacy of fungicides in the management of early blight of tomato (Alternaria solani). Int. J. of Plant Protection, 5 (2): 413-416. 